\title{
Active Control of Dynamic Bearing Loads in Rotating Machinery Using the Deflection Coefficient Method for Load Estimation
}

\author{
William W. Clark, Joo-Hyung Kim, Roy D. Marangoni \\ Vibration and Control Laboratory, Mechanical Engineering Department, \\ University of Pittsburgh, Pittsburgh, Pennsylvania, USA
}

\begin{abstract}
A new technique is presented for measuring and actively controlling dynamic bearing loads in rotating machinery subject to periodic excitations. Bearing loads are estimated using the Deflection-Coefficient Method, a technique which does not rely on a full system model, and which applies commonly-used shaft-deflection measurement equipment to obtain estimates of bearing loads. The estimated bearing load is used as an error signal in an adaptive feedforward disturbance rejection controller. The result is a control system which can selectively minimize dynamic bearing loads in real time in rotating machinery systems. The method is applied to a numerical model of a typical rotating machinery system to suppress dynamic reaction forces at bearing supports, and then it is experimentally verified on a beam test rig.
\end{abstract}

\section{INTRODUCTION}

The availability of powerful computing equipment and precision actuators and sensors has led to an increased interest in the use of active control techniques for suppressing vibrations in rotating machinery. Much of that effort has focused on suppressing shaft displacements [Burrows et al., 1989, Stanway and Burrows, 1981, Ulsoy, 1984], and for good reason, since a primary objective in rotating machinery operation is to prevent interference between rotating and stationary components. An issue that is often seen as secondary is that of dynamic loads in bearings which are a result of the vibrations. Prolonged exposure to excessive loads can lead to reductions in bearing life, and ultimately increased maintenance and down-time for machinery [Collacott, 1977, Bradfield et al., 1991]. For that reason, suppression of bearing loads can be just as important to the life of a rotating machine as can controlling vibration amplitudes.

Active control of bearing loads has seen little attention in the literature for two primary reasons. Most importantly, bearing loads are very difficult to measure, especially as compared to shaft deflections, and without measurement they are difficult to control. Second, the general line of reasoning used in much of the literature has been, then, that by controlling shaft deflections, dynamic bearing loads will be held in check. Recent studies have shown, however, that decreased shaft deflections do not necessarily coincide with decreased bearing loads, and can, in fact, result in increased loads [Clark et al., 1993, Kim et al., 1993].

This paper presents a method for actively controlling dynamic bearing loads in a rotating machine subject to periodic excitations. At the heart of the control process is a method for estimating bearing loads, herein called the Deflection-Coefficient Method [Marangoni, 1990]. The method relies on standard rotating machinery displacement sensors and beam theory to infer the dynamic loads in a bearing support. No detailed model of the system is required, other than information about the shaft properties and its boundary conditions. The bearing load information is then incorporated into a feedforward vibration control method to minimize the reaction forces at the bearing. The result is a straightforward, non-invasive method which uses standard sensors and a common control approach that can be retrofitted to existing rotating machinery systems or incorporated into new systems.

The paper first presents the Deflection-Coefficient Method (DCM) for estimating bearing loads, which involves determining shear forces in the shaft from a set of closelyspaced deflection measurements. The technique is verified by comparing its results to the exact analytical reaction forces found in a simply-supported and a clamped-pinned beam. Next, the controller is presented. The control technique involves applying a controlling force to the system that cancels the effects of a disturbance which may arise from rotating unbalance or shaft misalignment. The magnitude and phase of the control force is adapted (using a Least Mean Square algorithm) so that an error signal, which in this case is the bearing load signal, is minimized.

After the bearing-load measurement and the control techniques are presented, the method is applied to a model of a rotating machinery system. A rotor-coupler-motor system is modeled as a flexible beam with lumped masses which vibrates in two uncoupled planes. To simplify the analysis, the control is applied in one plane only. Simulations are carried out to show the performance of this technique in minimizing dynamic reaction forces. Bearing loads are determined using the deflection-coefficient method, and the control force is generated to minimize that signal. The results show that the bearing loads can be greatly reduced using this technique, particularly when the first system mode dominates the response.

Finally, the technique is experimentally applied to a flexible beam test rig to suppress the dynamic reaction forces at the supports. 